Feltes TF, eds. Moss and Adams's Heart Disease in Infants, Children, and Adolescents. 7th ed. Philadelphia, PA: Lippincott \& Wilkins; 2008:761-92.

doi:10.1016/j.jtcvs.2009.11.004

\section{THE FUNDAMENTAL} IMPORTANCE OF BASELINE COMPARISONS IN A CLINICAL TRIAL

\section{To the Editor:}

We read with interest the study by Hecht-Dolnik and associates in the September 2009 issue of the Journal, " $\mathrm{He}$ tastarch Increases the Risk of Bleeding Complications in Patients After OffPump Coronary Bypass Surgery: A Randomized Clinical Trial." In this article, they conclude that the intraoperative administration of $1 \mathrm{~L}$ of hetastarch was associated with an increase in postoperative chest tube drainage and transfusion of blood products, which resulted in early termination of the study.

Although we acknowledge the importance of the clinical question the authors have addressed, we have major concerns regarding the equality of the intervention and the control groups at baseline and the method in which this is reported. Although the authors claim that there were no statistically significant differences in baseline characteristics between groups, Table 1 indicates the baseline international normalized ratio (INR) in the hetastarch group was 1.47 (2.12) whereas in the albumin group baseline INR was 1.07 (0.10), values presented as mean (standard deviation). Although the mean value between these 2 groups may not be statistically different, it is misleading to claim baseline equivalence between the groups. The upper limit of INR is typically quoted at 1.3 , indicating the mean INR in the hetastarch group is abnormal and in the albumin group mean INR is normal. Furthermore, the large standard deviation in baseline INR in the hetastarch group can only be explained by the presence of one or more outliers with markedly abnormal baseline value. In contrast, the standard deviation for INR of the albumin group is small (0.10), indicating a typically normal distribution. We would suggest that this method of reporting baseline INR is potentially misleading. Presenting the proportion of patients in each group with abnormal INR at baseline would be substantially more informative to judge baseline equivalence of the 2 groups.

This difference in baseline INR and, by inference, bleeding risk is difficult to explain in a trial whose group assignment was by random allocation. However, in a study whose primary outcome was transfusion of blood products, understanding a difference of this nature is vitally important to interpretation of the results, particularly where transfusion practice was neither blinded nor protocol guided, as in this study.

We believe this highlights the importance of using the appropriate method of data presentation to describe baseline characteristics in a clinical trial as well as the potential pitfall of relying on $P$ values greater than .05 to indicate baseline equivalence of groups rather than clinical interpretation for the potential influence of an observed difference on the outcome of interest.

David McIlroy, MD, MClinEpi, FANZCA Ervant Nishanian, $M D, P h D$ Department of Anesthesiology Columbia University College of Physicians and Surgeons New York, NY

doi:10.1016/j.jtcvs.2009.10.058

\section{Reply to the Editor:}

We extend our thanks to Drs McIlroy and Nishanian for their careful reading of our article. Their concerns would be well placed if the average baseline international normalized ratio (INR) in the hetastarch group were 1.47. Their comment led us to review the project source data. That review revealed several transcription errors in which the decimal place in the INR was misplaced. The average baseline INR after correction of those transcription errors is 1.06 (standard deviation $=0.11$ ). This correction thus reverses the direction of the difference between the hetastarch and albumin groups in the average INR from that presented in the initial article. The statistical significance of the difference in average INR between the albumin and hetastarch groups becomes even weaker, remaining not statistically significant (Student $t=0.77 ; P=.44$, not significant).

We then investigated how many cases in each group had a baseline INR above the 1.30 threshold, as Drs McIlroy and Nishanian suggested. That threshold is crossed by 2 members $(2.6 \%)$ of the albumin group and 3 members $(3.8 \%)$ of the hetastarch group. The difference in the proportion in each group with an INR above the 1.3 threshold is not statistically significant (Fisher exact test $=1.0$, not significant).

This corrected finding supports treating the intervention and control groups in this study as equivalent. We apologize for any misunderstandings caused by this inaccuracy in the initial reported statistics summarizing and comparing baseline characteristics.

Marketa Hecht-Dolnik, $M D^{a}$
Howard Barkan, DrPH
Ananse Taharka, $M D^{a}$
John Loftus, $M D^{a}$
${ }^{a}$ Kaiser Permanente Medical Center
Oakland, Calif
${ }^{b}$ University of California
Berkeley, Calif

doi:10.1016/j.jtcvs.2009.11.031

\section{TECHNICAL OPTIONS FOR THE TREATMENT OF ANOMALOUS ORIGINS OF RIGHT OR LEFT CORONARY ARTERIES \\ ASSOCIATED WITH AORTOPULMONARY WINDOWS}

\section{To the Editor:}

Aortopulmonary window (APW) and anomalous right coronary artery (RCA) originating from the pulmonary 